\title{
EFICIENCIA Y PRODUCTIVIDAD DE LOS SISTEMAS DE SALUD DE LOS PAÍSES DE LA UNIÓN EUROPEA.
}

\author{
JUAN CÁNDIDO GÓMEZ GALLEGO \\ Departamento de Economía Financiera y Contabilidad, UNIVERSIDAD CATÓLICA DE MURCIA, ESPAÑA. \\ e-mail: jcandido@ucam.edu \\ JAVIER FERNÁNDO GARCÍA GARCÍA \\ Departamento Ciencias de la Salud, UNIVERSIDAD CATÓLICA DE MURCIA, ESPAÑA. \\ e-mail: fggar@gmail.com \\ MARÍA GÓMEZ GALLEGO \\ Departamento Ciencias de la Salud, UNIVERSIDAD CATÓLICA DE MURCIA, ESPAÑA. \\ e-mail: mggallego@ucam.edu
}

\section{RESUMEN}

En el presente trabajo se evalúa y analiza la eficiencia técnica en la gestión de los sistemas de salud de 28 países de la Unión Europea. Tal análisis, supone la estimación de la eficiencia técnica, el cálculo de la cross-eficiencia y la valoración del cambio en productividad entre los años 2012 a 2015. Los datos proceden de la base on line Eurostat. Se aplica el DEA-bootstrap y se calculan los índices de Malmquist. Los resultados afirman que los países de la UE han experimentado un pequeño crecimiento de la productividad en los sistemas de salud en el periodo 2012-2015, que se asocia a un cambio positivo en la eficiencia, pero no a mejoras en la tecnología. Son necesarios estudios adicionales que investiguen sobre qué factores observables o latentes de los países sirven para explicar los niveles de productividad de los sistemas de salud de los países de la Unión Europea.

Palabras clave: Sistemas de salud, eficiencia, DEA-bootstrap, índices de Malmquist.

\section{Efficiency and productivity of the health systems of the countries of the European Union.}

\begin{abstract}
In this paper the technical efficiency of the health systems of 28 UE countries is analyzed. Such analysis assumes the cross-efficiency calculations and the valuation of productivity changes between the years 2012-2015. The source of data is the Eurostat online database. DEAbootstrap is applied and Malmquist index are calculated. The results appears co confirm that EU countries have suffered a small increase in productivity in HS, which is related with positive changes in efficiency but not in technology. Even so, further research should be necessary to find the latent factors to explain the productivity levels of the HS in EU.
\end{abstract}

Keywords: Health systems, efficiency, DEA-bootstrap, Malmquist index

Clasificación JEL: C14, I12 


\section{INTRODUCCIÓN}

En los últimos años se viene produciendo, en la mayoría de los países, un incremento considerable del gasto en salud ${ }^{1}$. Este es un tema abordado con preocupación por todos los gobiernos, debido, además, a los considerables efectos que tiene tal volumen de gasto sobre la economía. Algunos estudios señalan entre las posibles causas del importante crecimiento del gasto el uso de mayor cantidad de recursos médicos por persona (Antoñanzas et al., 2016), y otros trabajos lo asocian a los cambios demográficos en la estructura de la población (Sorenson et al., 2013). Además, el problema se ha agravado en la reciente crisis económica; los recortes en los recursos financieros experimentados por la mayoría de los países europeos han reducido las fuentes disponibles para el mantenimiento y desarrollo de las políticas sanitarias (Nuti et al., 2011; Quaglio et al., 2013). Y, sin embargo, las expectativas y requerimientos de los ciudadanos sobre los servicios de salud continúan incrementándose (Saurina et al., 2012).

Ante esta situación, resulta fundamental desarrollar e implementar líneas estratégicas dirigidas a evitar el malgasto por ineficiencias y, en consecuencia, a la maximización de la productividad de los sistemas sanitarios (González et al., 2010; Mirzosaid, 2011) y así, poder hacer frente a la financiación de los requeridos programas de protección de la salud (Georgiou, 2010).

Existen algunos estudios sobre la medición de la eficiencia de los sistemas de salud en países europeos. Una revisión sistemática y de meta análisis es Varabyova y Müller (2016). Otros trabajos de interés son: Afonso yAubyn, 2005, 2011; Asandului et al., 2014; Gearhart, 2016; González et al., 2010; Hernández y Moral-Benito, 2014; Moreno-Enguix et al., 2017; Pérez-Cárceles et al., 2017; RetzlaffRoberts et al., 2004; Spinks y Hollingsworth, 2009).

En lo que se refiere a la metodología, uno de los métodos más utilizados para valorar la eficiencia es el Análisis Envolvente de Datos, DEA, que calcula la eficiencia relativa de múltiples unidades de decisión que operan con objetivos similares (Farrell, 1957; Charnes et al., 1978). Los primeros trabajos que presentan aproximaciones analíticas para medir la eficiencia de producción son los desarrollados por Koopmans (1951), Debreu (1951) y Farrell (1957), así como los modelos y funciones distancia de Shephard (Shephard, 1953, 1970). Los trabajos de Shephard (1953, 1970), Koopmans (1951) aplican modelos de programación microeconómica para realizar análisis de eficiencia y productividad estableciendo el supuesto de convexidad sobre la tecnología en DEA. Charnes, Cooper y Rhodes (1978) realizaron importantes aportaciones a la teoría y popularizaron la aplicación del modelo DEA-CCR. Entre las hipótesis establecidas para el modelo DEA-CCR se encuentran la de convexidad y rendimientos constantes a escala. La relajación de esta última, al permitir rendimientos variables a escala en la tecnología de producción, da lugar al denominado modelo DEA-BBC de Banker et al., (1984).

El procedimiento DEA, tal y como lo plantean los autores, adolece de una conocida debilidad que es su carácter de autoevaluación, asigna como ponderaciones a inputs y outputs para cada unidad, aquellas que le son más favorables. Para resolver este problema se introduce el concepto de cross-efficiency que evalúa la eficiencia de cada unidad desde la perspectiva de cada una de las demás unidades, se construye una matriz de eficiencias cruzadas que puede ser evaluada con la aplicación de metodologías multivariantes (Doyle \& Green, 1994; Liang, et al., 2008). Pues bien, ante el problema real planteado sobre financiación de los sistemas de salud y en la era de los objetivos de desarrollo sostenible y en particular el avance hacia la cobertura universal de ssalud imperativo reducir el desperdicio de recursos para garantizar el acceso sostenible de la población a los servicios de salud necesarios y efectivos, sin soportar las dificultades financieras. Así, un objetivo prioritario de los gobiernos es mejorar en los niveles de eficiencia en la gestión de sus sistemas sanitarios y, así, incrementar también la productividad de los mismos.

El objetivo de este estudio es evaluar en el periodo 2012 a 2015 la eficiencia técnica y el cambio total en la productividad de los factores de los sistemas nacionales de salud de 28 países en UE.

La estructura del trabajo es la siguiente: En el apartado 2 se exponen los procedimientos metodológicos aplicados en el estudio (DEA, cross-eficiencia e índices de Malmquist). El apartado 3 contiene los resultados. A continuación, se exponen las conclusiones $\mathrm{y}$, por último, las referencias citadas en el texto.

\footnotetext{
${ }^{1}$ Según Eurostat, en los países europeos el gasto en salud como porcentaje del PIB ha pasado del 7,92 en 2012 al 8,40\% en el 2015 , por término medio.
} 


\section{MATERIAL Y MÉTODOS}

\subsection{EI modelo DEA}

El trabajo de Farrell (1957) establece las bases metodológicas que permiten a Charnes, Cooper y Rhodes desarrollar unos modelos matemáticos basados en técnicas de programación, capaces de estimar la eficiencia técnica con la que opera una muestra de unidades productivas, DMUs. El modelo de Charnes, Cooper y Rhodes o modelo CCR (1978) permite evaluar la eficiencia de una muestra de entidades caracterizadas por una función de producción multiproducto que emplea varios inputs. Este indicador de eficiencia se define como el cociente de la suma ponderada de outputs entre la suma ponderada de los inputs. El modelo se formula como un problema de optimización matemática condicionada, en el que las variables que se deben calcular son el propio indicador de eficiencia y las ponderaciones asociadas a las variables inputs y outputs que caracterizan a las unidades productivas. La formulación matemática del modelo CCR, en su versión fraccional para el caso de $n$ unidades productivas que producen $\mathrm{s}$ outputs a partir de $\mathrm{m}$ inputs, es la siguiente:

$$
\operatorname{Max}_{0}=\frac{\sum_{r=1}^{S} u_{r 0} y_{r 0}}{\sum_{i=1}^{m} v_{i 0} x_{i 0}}
$$

s.a.

$$
\begin{array}{cl}
\frac{\sum_{r=1}^{s} u_{r j} y_{r j}}{\sum_{i=1}^{m} v_{i j} x_{i j}} \leq 1, & \quad 1 \leq j \leq n \\
u_{r 0} \geq 0, & 0 \leq r \leq s \\
v_{i 0} \geq 0 & 0 \leq i \leq m
\end{array}
$$

La unidad productiva cuya eficiencia se va a calcular se denota por el subíndice 0 , las variables $y_{r j}$ y $x_{i j}$ representan, respectivamente, las cantidades de output $\mathrm{r}$ y de input $\mathrm{i}$ de la entidad $\mathrm{j}$. Finalmente, $v_{r 0} \mathrm{y} u_{i 0}$ representan las ponderaciones atribuidas al output $\mathrm{r}$ y al input i respectivamente y que corresponden a la entidad cuya eficiencia se va a evaluar. La resolución de este programa matemático permite determinar los valores de las variables $u_{r 0}$ y $v_{i 0} \mathrm{y}$, por tanto, el índice de eficiencia E0 asignado a la unidad productiva evaluada. Resolviendo este programa para cada una de las $\mathrm{n}$ entidades, se obtiene la medida de la eficiencia escalar para cada una de ellas. Las ponderaciones asignadas a las variables inputs y outputs se determinan endógenamente por la resolución del modelo y pueden variar entre las distintas unidades productivas. Estas ponderaciones deben $\operatorname{ser}^{2}$ mayores o iguales a cero. En general, una entidad dará mayor peso a los inputs que utilicen menos y a los outputs que produce en una cantidad mayor ya que la ponderación que se obtiene al resolver el problema se calcula de tal forma que ofrece a cada DMU la valoración en términos de eficiencia más favorable posible (Charnes et al., 1978). La complejidad en la resolución de los problemas de programación en su versión fraccional conduce a Charnes, Cooper y Rhodes a convertirlos en problemas equivalentes de programación lineal. El modelo lineal equivalente conocido como CCR lineal con orientación input (se maximiza el numerador manteniendo fijo el denominador), se formula de la forma siguiente:

$$
\begin{gathered}
\operatorname{Max}_{0}=\sum_{r=1}^{s} u_{r 0} y_{r 0} \\
\text { s.a. } \\
\sum_{r=1}^{s} u_{r 0} y_{r j}-\sum_{i=1}^{m} v_{i 0} x_{i j} \leq 0, \quad 1 \leq j \leq n
\end{gathered}
$$

\footnotetext{
${ }^{2}$ Hay que señalar que la DMU objeto de evaluación aparece tanto en la función objetivo como en la restricción, lo que garantiza que siempre existe una solución al problema de programación matemática, con un valor de la función objetivo acotado entre 0 y 1 (Lewin y Morey, 1981).
} 


$$
\begin{gathered}
\sum_{i=1}^{m} v_{i 0} x_{i 0}=1 \\
u_{r 0}>\varepsilon, \quad 0 \leq r \leq s \\
v_{i 0}>\varepsilon \quad 0 \leq i \leq m
\end{gathered}
$$

La variable $\phi_{0}$ representa el índice de eficiencia obtenido con una orientación input. Como cualquier programa lineal, el anterior problema de programación tiene asociado un problema problemas dual (Cooper et al., 2007). La formulación del problema dual asociado al anterior problema primal CCR, en su versión lineal y con una orientación input es la siguiente:

$$
\operatorname{Min} \theta_{0}-\left(\sum_{i=1}^{m} s_{i}^{+}-\sum_{r=1}^{s} s_{r}^{-}\right)
$$

s.a.

$$
\begin{gathered}
\sum_{j=1}^{n} \lambda_{j} x_{i j+} s_{i}^{+}=\theta_{0} x_{i 0}, \quad i=1, \ldots, m \\
\sum_{j=1}^{n} \lambda_{j} y_{r j-} s_{r}^{-}=y_{r 0}, \quad r=1, \ldots, s \\
\lambda_{j} \geq 0 \\
s_{i}^{+} \geq 0 \\
s_{r}^{-} \geq 0
\end{gathered}
$$

La variable $\theta_{0}$ representa el ratio de eficiencia de la unidad productiva cuya eficiencia se está evaluando. Las variables $\lambda_{\mathrm{j}}$, constituyen los parámetros a partir de los que se construye el grupo de referencia de la unidad productiva objeto de evaluación y constituyen, junto a $\theta_{0}$ las variables que se tienen que calcular en el modelo. Las variables $\mathrm{s}_{\mathrm{i}}^{+}$y $\mathrm{s}_{\mathrm{r}}^{-}$son la holgura de cada una de las $\mathrm{m}+\mathrm{s}$ restricciones del modelo.

Como señalan Charnes, Cooper y Rhodes (1978), la eficiencia de una unidad productiva exige la concurrencia simultánea de dos condiciones: el índice de eficiencia debe ser igual a uno y las variables de holgura de todos los inputs y los outputs deben ser nulas, esto es, $\mathrm{s}_{\mathrm{i}}^{+}=0$ y s $\mathrm{s}_{\mathrm{i}}^{-}=0$. En este caso, no existe en la muestra ninguna otra unidad productiva que consuma, como mucho, los mismos recursos que la DMU0 y produzca, al menos, lo mismo que ella. Por consiguiente, todas las variables $\lambda_{\mathrm{j}}$ serán nulas, excepto la correspondiente a la propia entidad que evaluamos, $\lambda_{0}$ que valdrá uno.

Una segunda posibilidad consiste en que la unidad productiva DMU0 sea ineficiente. En este caso $\lambda_{j}>0$ con $j \neq 0$. Por consiguiente, en la muestra existe alguna entidad que consume como mucho los mismos recursos que la DMU0 y produce al menos lo mismo que ella. Además, la ineficiencia de la unidad evaluada se puede caracterizar hasta de tres formas diferentes:

En primer lugar, la unidad productiva evaluada puede obtener un índice de eficiencia unitario y no satura las restricciones (alguna de las variables de holgura es distinta de 0). Esto indica que no es posible ningún incremento (reducción) radial de los outputs (inputs), pero sí un incremento (reducción) específico y no radial de algún output (input).

En segundo lugar, el índice de eficiencia es menor de uno y todas las variables de holgura son nulas. En este caso, la unidad productiva no es eficiente, en la medida en que es posible obtener un incremento radial en todos sus outputs sin alterar los recursos que utiliza. 
Y una última posibilidad viene caracterizada cuando se obtiene un índice de eficiencia no unitario y alguna variable de holgura es positiva. En este caso, la eficiencia de la DMU0 exige primero un incremento radial en todos sus outputs en la proporción que determina el índice de eficiencia y, seguidamente, un incremento (disminución) específico y no radial del output (input) en el que aparece la holgura no nula.

Por consiguiente, combinando las variables de holgura con los resultados del índice de eficiencia podemos obtener un resultado global respecto del ahorro potencial en input y el incremento potencial en outputs que resulta de enorme interés desde una perspectiva de gestión.

\subsubsection{Ampliación rendimientos de escala: Modelo DEA BCC}

A un mayor nivel de producción o escala de operaciones puede verificarse que la unidad productiva exhiba un mayor nivel de eficiencia, derivado del aprovechamiento de las economías de escala. Cuando el modelo se especifica con rendimientos constantes a escala, se está obviando la influencia que la escala concreta en que opera una organización puede tener sobre sus posibilidades de producción. Para detectar las potenciales fuentes de ineficiencia, que provienen de la escala de operaciones en la que opera una entidad, se requiere formular un modelo como el que plantean Banker, Charnes y Cooper (1984) (BCC), caracterizando una función de producción con rendimientos variables a escala. La formulación matemática de un modelo BCC se obtiene añadiendo a la del modelo DEA CCR la condición $\sum_{j=1}^{n} \lambda_{j}=1$.

El modelo BCC permite obtener una cuantificación de la eficiencia técnica pura, no libre de los efectos de la escala en la que opera cada entidad. Por tanto, este modelo permite separar la eficiencia técnica y la eficiencia de escala de las unidades productivas.

\subsection{Sobre el sistema de ponderaciones en el DEA}

El Análisis Envolvente de Datos proporciona una medida de eficiencia relativa para unidades de toma de decisiones con múltiples inputs y outputs. Si bien se ha demostrado que el DEA es un enfoque eficaz para identificar fronteras de las mejores prácticas, se ha criticado su flexibilidad para ponderar los múltiples insumos y productos y su naturaleza de autoevaluación. Un objetivo de esta investigación es obtener medidas de eficiencia que no estén basadas en este criterio, sino que tengan en cuenta otras perspectivas en el empleo de inputs y de outputs, sin recurrir a restricciones sobre los pesos a partir de opiniones de los expertos del área de aplicación (Anderson et al., 2002).

El método que aplicamos parte del concepto de eficiencia cruzada (cross-efficiency) que se desarrolla como una extensión del DEA para clasificar DMU (Sexton et al., 1986). Se basa en la siguiente idea, una vez que se ha resuelto el modelo DEA, en el que se ha obtenido el mejor conjunto de pesos para una DMU0 particular, utilizar ese conjunto de pesos para ponderar los inputs y outputs de cada una de las otras DMUs y calcular la eficiencia cruzada de cada una de las otras DMUs desde el punto de vista de la DMU0 evaluada (original). El procedimiento se puede repetir para todas las DMUs, y así completar una matriz de eficiencias cruzadas. Las puntuaciones originales de la eficiencia de cada DMU constituyen la diagonal principal de la matriz.

Siguiendo la notación adoptada en este trabajo, sean $\mathrm{n}$ DMUs que consumen $\mathrm{m}$ inputs y producen $\mathrm{s}$ outputs. Sean $(\mathrm{i}=1,2, \ldots, \mathrm{m}),(\mathrm{r}=1,2, \ldots, \mathrm{s})$ los valores respectivos de inputs y outputs de la unidad $\mathrm{j}$ $(\mathrm{j}=1,2, \ldots, \mathrm{n})$.

Entonces, la eficiencia relativa $E_{k k}$ de la DMU k, se puede determinar mediante el modelo DEACCR:

$$
E_{k k}=\frac{\sum_{r=1}^{s} u_{r k} y_{r k}}{\sum_{i=1}^{m} v_{i k} x_{i k}}
$$

$E_{k k}$ denota la eficiencia que la DMUk puede lograr usando sus propios pesos.

Ahora,

$$
E_{j k}=\frac{\sum_{r=1}^{s} u_{r k} y_{r j}}{\sum_{i=1}^{m} v_{i k} x_{i j}}
$$

es el valor de la eficiencia cruzada de la DMU j, a partir de los de la DMUk. 
El promedio de los valores de la columna $\mathrm{k}$ se puede interpretar como una puntuación promedio dada a los pares por la DMU k (ya que se valora la eficiencia de las otras DMUs utilizando los pesos obtenidos para la DMU k). Calculamos

$$
\overline{\bar{E}}_{\boldsymbol{k}}=\frac{1}{n-1} \sum_{j=1, j \neq k}^{n} E_{j k}, \quad 0 \quad k=1,2, \ldots, n .
$$

dónde este promedio se puede hacer con o sin los elementos de la diagonal, es decir, utilizando o no, para hallar el valor medio, la propia eficiencia de la DMUk.

El promedio de las puntuaciones de la fila j podría ser considerado como la valoración promedio de la unidad $\mathrm{j}$ cuando es evaluada desde la perspectiva de cada DMUk.

$$
\bar{E}_{j}=\frac{1}{n-1} \sum_{k=1, k \neq j}^{n} E_{j k}, \quad 0 \quad j=1,2, \ldots, n .
$$

Varios aspectos a tener en cuenta: por un lado, el conjunto de referencia de la unidad evaluada parece más lógico considerar el conjunto de ponderaciones de las unidades "conjunto de referencia" de la DMU evaluada. Por otra parte, tener en cuenta la orientación productiva y, por supuesto, no considerar aquellas unidades que claramente son atípicas e independientes.

\subsection{Análisis de productividad. Índices de Malmquist}

Existen numerosos métodos para medir el crecimiento de la productividad. La elección entre ellos dependerá del objetivo que se desee obtener a través de la medición de la productividad y, en muchos casos, de la disponibilidad de los datos. En este trabajo aplicamos una conocida medida de productividad multifactorial, el índice de Malmquist.

La formulación de este índice fue introducida por Caves et al. (1982) y mejorada posteriormente por Fare et al. (1992). El procedimiento no requiere conocer los precios de los diferentes inputs y outputs, ni asumir ningún supuesto acerca del comportamiento de los productores, pero sí exige la estimación de una representación de la tecnología de producción.

Basándonos en la definición de la función distancia de Shephard orientada al output,

$$
D_{0}(x, y)=\max \{\theta: \theta y \in P(x)\}
$$

el cálculo del índice de productividad de Malmquist (MPI) requiere considerar dos períodos de tiempo distintos $(\mathrm{t} \text { y } \mathrm{t}+1)^{3}$. Así, si se considera que la referencia tecnológica es la tecnología en $\mathrm{t}$,

$$
\operatorname{MPI}_{C}^{t}\left(x^{t}, y^{t}, x^{t+1}, y^{t+1}\right)=\frac{D_{C}^{t}\left(x^{t+1}, y^{t+1}\right)}{D_{C}^{t}\left(x^{t}, y^{t}\right)}
$$

Alternativamente, podría definirse un índice Malmquist, con la referencia tecnológica en el período $\mathrm{t}+1$, como:

$$
M P I_{C}^{t+1}\left(x^{t}, y^{t}, x^{t+1}, y^{t+1}\right)=\frac{D_{C}^{t+1}\left(x^{t+1}, y^{t+1}\right)}{D_{C}^{t+1}\left(x^{t}, y^{t}\right)}
$$

A su vez, Fare et al. (1994) define el índice de cambios en la productividad Malmquist como la media geométrica de dos índices de productividad Malmquist de tipo Caves.

El índice queda definido a través de la siguiente expresión:

$$
M P I_{C}^{t}\left(x^{t}, y^{t}, x^{t+1}, y^{t+1}\right)=\left[\frac{D_{C}^{t}\left(x^{t+1}, y^{t+1}\right)}{D_{C}^{t}\left(x^{t}, y^{t}\right)} * \frac{D_{C}^{t+1}\left(x^{t+1}, y^{t+1}\right)}{D_{C}^{t+1}\left(x^{t}, y^{t}\right)}\right]^{1 / 2}
$$

\footnotetext{
${ }^{3}$ El sufijo "C" indica que se están considerando rendimientos constantes de escala (CRS).
} 
El MPI puede tomar valores superiores a 1, lo que implica que ha existido un crecimiento en la productividad, valores iguales a 1, representativos de un estancamiento de los niveles de productividad, o valores inferiores a 1, en cuyo caso se considera que la productividad de las unidades evaluadas ha decrecido a lo largo del período estudiado.

Mediante el uso de este método se pueden identificar las dos causas principales que pueden explicar un cambio en los niveles de productividad de cada unidad (Grifell-Tajté y Lovell, 1997): el cambio en la eficiencia técnica (EC) (conocido habitualmente en la literatura como efecto "catching up"), que indica si las unidades evaluadas se acercan o se alejan de su correspondiente frontera de eficiencia entre los períodos evaluados, y el cambio tecnológico (TC), representado por la media geométrica de su magnitud, que aproxima en qué medida, las unidades que forman la frontera de eficiencia, han mejorado o empeorado su productividad entre los períodos estudiados Jacobs et al. (2006).

La descomposición más habitual del índice de Malmquist es la que proponen Färe et al. (1994):

$$
\begin{gathered}
M P I_{C}^{t}\left(x^{t}, y^{t}, x^{t+1}, y^{t+1}\right)=\frac{D_{C}^{t}\left(x^{t+1}, y^{t+1}\right)}{D_{C}^{t}\left(x^{t}, y^{t}\right)} *\left\{\left[\frac{D_{C}^{t}\left(x^{t+1}, y^{t+1}\right)}{D_{C}^{t+1}\left(x^{t+1}, y^{t+1}\right)} * \frac{D_{C}^{t}\left(x^{t}, y^{t}\right)}{D_{C}^{t+1}\left(x^{t}, y^{t}\right)}\right]\right\}^{1 / 2} \\
=E C\left(x^{t}, y^{t}, x^{t+1}, y^{t+1}\right) * T C\left(x^{t}, y^{t}, x^{t+1}, y^{t+1}\right)
\end{gathered}
$$

Esta descomposición está basada en el uso de una tecnología de producción con rendimientos constantes de escala. No obstante, esta definición puede generar problemas de consistencia cuando este supuesto no sea aplicable, es decir, si existen rendimientos de escala en la producción Balk (2001).

\subsection{Datos y variables}

Para la estimación y análisis de la eficiencia se han utilizado datos estadísticos de 28 países europeos para los años 2012 y 2015, extraídos de la base de datos en línea de Eurostat. La fuente garantiza la precisión y comparabilidad de los datos, que son dos propiedades requeridas en los modelos DEA.

La selección de variables y su número es crucial en el análisis de eficiencia. En este trabajo seleccionamos tres variables de producción: la esperanza de vida al nacer, la esperanza de vida ajustada por la salud y la tasa de supervivencia infantil, y tres variables de entrada: el gasto público en salud como porcentaje del PIB; el número de médicos y el número de camas curativas y de rehabilitación.

Según Eurostat, las variables outputs e inputs se definen de la siguiente forma:

- Esperanza de vida al nacer (LE). Se define como el número medio de años que una persona vivirá, dadas las condiciones de vida actuales. La LE se considera como uno de los indicadores más directos de la eficiencia de los sistemas de salud, Tudor et al., (2009). Además, está relacionada con variables socioeconómicas como la educación, género, nivel de ingresos y estado civil, Jaba et al. (2011).

- Esperanza de vida ajustada por la salud (HALE). Se define como el número de años que una persona vivirá sin discapacidad. Es un indicador aceptado como un factor de productividad. Aumentar el valor de HALE es uno de los objetivos constantes de las políticas de la UE. Además de beneficios sociales, aumentar el valor de HALE conllevaría una disminución de costes para el sistema de salud y aumentaría la productividad de los empleados.

- Tasa de mortalidad infantil (MR). Se define por la relación entre el número de muertes de niños menores de un año durante el año y el número de nacidos vivos en ese mismo año. El valor se expresa por 1000 nacidos vivos. De acuerdo con los requerimientos del DEA, utilizamos el "índice de supervivencia infantil" (SR) que representa el número de niños que viven por cada uno que muere ((1000-MR)/MR).

- Gasto en salud per cápita (HEpc). Se define como el porcentaje del PIB asignado a la atención de la salud. Es decir, el total de gastos corrientes y de capital que figuran en los presupuestos gubernamentales (centrales y locales), donaciones y fondos sociales de seguros de salud.

- Número de camas curativas y de rehabilitación de hospital (B). Se define como el número de camas de hospital que están disponibles para la atención de pacientes ingresados por cada 10.000 habitantes. El total de camas de hospital incluye a las camas de agudos, camas de atención psiquiátrica; camas de cuidados prolongados y otras camas de hospital. 
- Número de médicos (P. Se define como número de personas que tienen un título licenciado en medicina y que tienen licencia para ejercer; médicos residentes, médicos asalariados, estatuarios, funcionarios y autónomos que prestan servicio, independientemente del lugar de prestación del servicio. El indicador se expresa por 10.000 habitantes.

\section{RESULTADOS}

La Tabla 1 muestra los estadísticos descriptivos de los inputs y outputs seleccionados en este estudio, para los años 2012 y 2015. También se muestran las correlaciones entre las variables.

Como muestra la Tabla 1, los promedios de inputs y outputs son valores representativos de las respectivas distribuciones (coeficiente de variación menor que 0,3). La variación media entre 2012 y 2015, dada por el promedio de las tasas de variación (anexos 1 y 2) es negativa para la variable HEgdp $\mathrm{y}$, las tasas de variación son positivas para $\mathrm{P}(4,53 \%)$ y para $\mathrm{B}(2,78 \%)$. Es decir, en lo que respecta a inputs no se ha mantenido la inversión en salud en términos relativos pero sí se han incrementado en términos absolutos los recursos humanos y materiales. Sin embargo, los promedios de las tasas de variación no son representativos, existe una alta variabilidad entre los 28 países. En cuanto al gasto en salud, 12 países tienen una tasa de variación negativa y 16 países incrementan el HEgdp entre 2012 y 2015. Por ejemplo, Bulgaria y Croacia incrementan el gasto en salud en un porcentaje superior al $8 \%$, mientras que Grecia lo disminuye en un porcentaje superior al 8\% y Eslovaquia lo hace en un porcentaje superior al 10\%. En este subgrupo destaca el caso de Irlanda, que disminuye el porcentaje de PIB dedicado al sistema de salud en un porcentaje del 31,13\%. España se encuentra en el grupo de países que incrementan en un porcentaje mínimo $(0,38 \%)$ el gasto público en salud.

Tabla 1.

Estadísticos descriptivos de inputs y output. Correlaciones de Pearson

\begin{tabular}{|c|c|c|c|c|c|c|c|}
\hline Año & Estadístico & $H E_{g d p}$ & $\mathbf{P}$ & B & LE & MR & HALE \\
\hline \multirow{5}{*}{2012} & Max. & 11,31 & 592,00 & 421,40 & 82,50 & 9,00 & 66,10 \\
\hline & Min. & 4,72 & 223,38 & 154,37 & 74,10 & 1,60 & 55,29 \\
\hline & Media & 8,35 & 344,10 & 269,71 & 79,21 & 3,95 & 61,78 \\
\hline & SD & 1,91 & 78,25 & 78,68 & 2,81 & 1,63 & 2,72 \\
\hline & C. Var. & 0,23 & 0,23 & 0,29 & 0,04 & 0,41 & 0,04 \\
\hline \multirow{5}{*}{2015} & Max. & 11,48 & 610,00 & 441,96 & 83,00 & 7,60 & 65,78 \\
\hline & Min. & 4,94 & 232,81 & 163,59 & 74,60 & 1,60 & 55,08 \\
\hline & Media & 8,24 & 358,86 & 276,80 & 79,74 & 3,65 & 61,38 \\
\hline & SD & 1,85 & 78,78 & 81,22 & 2,79 & 1,34 & 3,05 \\
\hline & C. Var. & 0,22 & 0,22 & 0,29 & 0,04 & 0,37 & 0,05 \\
\hline \multicolumn{2}{|c|}{ Tasa variación media } & $-0,684$ & 4,535 & 2,787 & 0,669 & 8,859 & $-0,935$ \\
\hline \multicolumn{8}{|c|}{ Correlaciones de Pearson } \\
\hline & & HEgdp & $\mathbf{P}$ & B & LE & MR & HALE \\
\hline & HEgdp & 1 & $0,239^{* * *}$ & $0,283^{* * *}$ & $0,662^{* * *}$ & $0,288^{* * *}$ & $0,281^{* * *}$ \\
\hline & $\mathrm{P}$ & & 1 & 0,023 & $0,153^{* *}$ & 0,018 & 0,115 \\
\hline & $\mathrm{B}$ & & & 1 & $0,559 * * *$ & $0,291^{* * *}$ & $0,227^{* * *}$ \\
\hline & LE & & & & 1 & $0,500^{* * *}$ & $0,439 * * *$ \\
\hline & ISR & & & & & 1 & 0,107 \\
\hline
\end{tabular}

Fuente: Elaboración propia a partir de datos de Eurostat para 2012 y 2015.

Nota: ${ }^{* * *}($ sig. $<0,01) ;{ }^{* *}($ sig. $<0,05)$ 
El comportamiento de los países en las variables $\mathrm{P}$ y $\mathrm{B}$ es más homogéneo. El número de médicos se incrementa en el periodo en todos los países a excepción de Italia ( $t v=-0,91 \%)$. En España se mantiene estable el número de profesionales, ya que se incremente en un $0,57 \%$. El número de camas también aumenta en el periodo para la mayoría de países (22) con un máximo en la tasa de variación de Dinamarca (23,35\%). En España baja el número de camas por habitante en un porcentaje del 1,24\%.

En el caso de los outputs el comportamiento es diferente para las tres variables. La esperanza de vida aumenta en los 28 países con una tasa media de $0,67 \%$ y con valores más altos en Estonia $(1,69 \%)$ y Finlandia $(1,12 \%)$. España incrementa la esperanza de vida en 0,5 años, un $0,61 \%$, pero hay que destacar que es el país de la Unión Europea de mayor LE en los dos años considerados. El comportamiento de la ratio de supervivencia infantil es diferente. La tasa media de variación es alta $(8,86 \%)$ y positiva para la mayoría de los 28 países. Con respecto a la esperanza de vida ajustada por la salud, hay que señalar que su evolución difiere al que ha tenido la esperanza de vida. Ahora, HALE presenta en 20 países una tasa de variación negativa, por ejemplo, Hungría $(-9,59 \%)$ y Suecia $(-8,24 \%)$; aumenta la esperanza de vida pero disminuye el número de años exentos de minusvalías.

Además, la tabla 1 muestra las correlaciones entre las seis variables (inputs y outputs). Los coeficientes de correlación tienen signo positivo, en todos los casos. Los tres inputs correlacionan significativamente con al menos un output. Merece especial atención el caso del input $\mathrm{P}$, que correlaciona con el output LE pero no con MR ni con HALE.

\subsection{Estimaciones de eficiencia técnica}

La tabla 2 contiene los resultados de las estimaciones de eficiencia con modelos de orientación inputs bajo rendimientos variables a escala y rendimientos constantes a escala. También se muestran las puntuaciones de eficiencia debido a la escala de operaciones. En una comparación global, cuando se considera CRS, ocho países resultan eficientes en ambos periodos, 2012 y 2015 (Bélgica, Bulgaria, Alemania, Lituania, Luxemburgo, Polonia, Rumanía y Eslovenia). En términos de promedios, el sistema europeo pasa de una eficiencia técnica de 0,874 a una de 0,888 , es decir, una pequeña mejora de 1,4 puntos porcentuales.

Cuando se establece VRS, en el año 2012 resultan 13 países eficientes y 15 que presentan X ineficiencias. En este grupo están Dinamarca, Grecia y Portugal. En el 2015, mejoran los resultados y aparecen 13 países con ineficiencias de gestión entre los que se incluyen Dinamarca, Portugal y Suecia como casos con mayores ineficiencias. Todos los países eficientes están en la frontera de producción y, además, tienen holguras nulas. En el año 2012, aparecen con mayor frecuencia como de países de referencia para otros países ineficientes: Luxemburgo (referencia de 13 países), Lituania (referencia de 6 países), Rumanía (referencia de 6 países), Alemania (referencia de 3 países) y Austria (referencia de 3 países).La situación es muy parecida en el 2015:Luxemburgo (referencia de 12 países), Lituania (referencia de 4 países), Polonia (referencia de 7 países) y Alemania (referencia de 2 países), ver anexos 3 y 4.

Como se ha comentado en el apartado de material y métodos, una debilidad del DEA es su carácter de autoevaluación, ya que asigna a inputs y outputs, en cada DMU evaluada, los pesos que más le favorecen; sin que necesariamente tal criterio responda al óptimo deseable desde otras perspectivas técnicas o sociales. Así, un país como Bulgaria podría asignar todo el peso a "Camas" o "Médicos" si tales recursos le resultaran de bajo coste, y así resultar clasificada como eficiente. Tal debilidad del DEA tiene suficiente importancia y, por ello, lo abordamos en este trabajo mediante el cálculo de la matriz de eficiencias cruzadas, no mostrada. En la siguiente tabla 3 se muestran a modo de resumen, los valores medios de las puntuaciones de eficiencia obtenidas con los criterios de las demás DMUs. En la tabla 3 y gráfico 1 se puede observar el tamaño de la discrepancia entre las puntuaciones de autoevaluación y las obtenidas por término medio con los criterios de los pares de la DMU evaluada. Son especialmente llamativos los casos de cinco países donde existe una enorme disparidad entre criterios de ponderación propia con el promedio de las evaluaciones hechas con los criterios de los demás países. Entre paréntesis se expresa el número de veces que es superior la eficiencia con el DEA convencional (autoevaluación) a la obtenida como cross-eficiencia por varios países: Bulgaria $(22,27)$, Hungría $(16,59)$, Letonia $(36,63)$, Lituania $(15,01)$ y Rumanía $(73,52)$. Para el resto de los países el 
cociente oscila entre Luxemburgo $(1,02)$ y Eslovaquia $(4,14)$. En el caso de España el cociente es de 1,48 .

Tabla 2

Eficiencias según modelos con orientación inputs para los años 2012 y 2015

\begin{tabular}{|c|c|c|c|c|c|c|c|c|c|c|}
\hline \multirow[b]{3}{*}{ País } & \multicolumn{5}{|c|}{ Año 2012} & \multicolumn{5}{|c|}{ Año 2015} \\
\hline & \multicolumn{3}{|c|}{ VRS } & \multirow[t]{2}{*}{ ECRS } & \multirow[t]{2}{*}{ EE } & \multicolumn{3}{|c|}{ VRS } & \multirow[t]{2}{*}{ ECRS } & \multirow[t]{2}{*}{ EE } \\
\hline & EVRS & LI & LS & & & EVRS & LI & LS & & \\
\hline Austria & 1,000 & 1,000 & 1,000 & 1,000 & 1,000 & 1,000 & 1,000 & 1,000 & 0,982 & 0,982 \\
\hline Bélgica & 1,000 & 1,000 & 1,000 & 1,000 & 1,000 & 1,000 & 1,000 & 1,000 & 1,000 & 1,000 \\
\hline Bulgaria & 1,000 & 1,000 & 1,000 & 1,000 & 1,000 & 1,000 & 1,000 & 1,000 & 1,000 & 1,000 \\
\hline Croacia & 0,893 & 0,785 & 0,954 & 0,892 & 1,000 & 0,859 & 0,717 & 0,891 & 0,847 & 0,987 \\
\hline Chipre & 0,967 & 0,934 & 1,000 & 0,911 & 0,942 & 0,931 & 0,861 & 1,000 & 0,930 & 1,000 \\
\hline R. Checa & 0,919 & 0,839 & 1,000 & 0,903 & 0,982 & 0,955 & 0,910 & 1,000 & 0,952 & 0,996 \\
\hline Dinamarca & 0,710 & 0,421 & 0,710 & 0,663 & 0,934 & 0,739 & 0,478 & 0,739 & 0,663 & 0,897 \\
\hline Estonia & 0,993 & 0,986 & 1,000 & 0,976 & 0,983 & 1,000 & 1,000 & 1,000 & 1,000 & 1,000 \\
\hline Finlandia & 0,855 & 0,709 & 0,872 & 0,798 & 0,934 & 1,000 & 1,000 & 1,000 & 0,863 & 0,863 \\
\hline Francia & 1,000 & 1,000 & 1,000 & 0,782 & 0,782 & 0,944 & 0,888 & 1,000 & 0,795 & 0,842 \\
\hline Alemania & 1,000 & 1,000 & 1,000 & 1,000 & 1,000 & 1,000 & 1,000 & 1,000 & 1,000 & 1,000 \\
\hline Grecia & 0,821 & 0,642 & 0,821 & 0,758 & 0,924 & 0,827 & 0,654 & 0,869 & 0,763 & 0,922 \\
\hline Hungría & 0,837 & 0,675 & 0,852 & 0,827 & 0,988 & 0,858 & 0,716 & 0,889 & 0,848 & 0,988 \\
\hline Irlanda & 0,984 & 0,969 & 1,000 & 0,877 & 0,891 & 0,974 & 0,948 & 1,000 & 0,890 & 0,913 \\
\hline Italia & 1,000 & 1,000 & 1,000 & 0,720 & 0,720 & 1,000 & 1,000 & 1,000 & 0,742 & 0,742 \\
\hline Letonia & 0,924 & 0,847 & 1,000 & 0,908 & 0,984 & 1,000 & 1,000 & 1,000 & 1,000 & 1,000 \\
\hline Lituania & 1,000 & 1,000 & 1,000 & 1,000 & 1,000 & 1,000 & 1,000 & 1,000 & 1,000 & 1,000 \\
\hline Luxemburgo & 1,000 & 1,000 & 1,000 & 1,000 & 1,000 & 1,000 & 1,000 & 1,000 & 1,000 & 1,000 \\
\hline Malta & 0,987 & 0,974 & 1,000 & 0,850 & 0,861 & 0,981 & 0,962 & 1,000 & 0,876 & 0,892 \\
\hline Países Bajos & 0,846 & 0,692 & 0,870 & 0,757 & 0,895 & 1,000 & 1,000 & 1,000 & 0,805 & 0,805 \\
\hline Polonia & 1,000 & 1,000 & 1,000 & 1,000 & 1,000 & 1,000 & 1,000 & 1,000 & 1,000 & 1,000 \\
\hline Portugal & 0,761 & 0,523 & 0,761 & 0,702 & 0,923 & 0,747 & 0,493 & 0,747 & 0,733 & 0,982 \\
\hline Rumania & 1,000 & 1,000 & 1,000 & 1,000 & 1,000 & 1,000 & 1,000 & 1,000 & 1,000 & 1,000 \\
\hline Eslovaquia & 0,923 & 0,845 & 1,000 & 0,917 & 0,994 & 0,936 & 0,872 & 1,000 & 0,927 & 0,990 \\
\hline Eslovenia & 1,000 & 1,000 & 1,000 & 1,000 & 1,000 & 1,000 & 1,000 & 1,000 & 1,000 & 1,000 \\
\hline España & 1,000 & 1,000 & 1,000 & 0,715 & 0,715 & 1,000 & 1,000 & 1,000 & 0,734 & 0,734 \\
\hline Suecia & 0,780 & 0,559 & 0,780 & 0,643 & 0,824 & 0,686 & 0,371 & 0,686 & 0,642 & 0,936 \\
\hline Reino Unido & 1,000 & 1,000 & 1,000 & 0,877 & 0,877 & 0,992 & 0,984 & 1,000 & 0,878 & 0,885 \\
\hline
\end{tabular}


Tabla 3

Medidas de cross-eficiencia en el 2015 (porcentajes).

\begin{tabular}{|c|c|c|c|c|c|c|c|}
\hline País & Austria & Bélgica & Bulgaria & Croacia & Chipre & Czechia & Dinamarca \\
\hline E CRS & 100,00 & 100,00 & 100,00 & 85,87 & 93,06 & 95,50 & 73,91 \\
\hline ECRS por pares & 64,64 & 75,46 & 4,49 & 28,54 & 74,66 & 51,33 & 46,48 \\
\hline País & Estonia & Finlandia & Francia & Alemania & Grecia & Hungria & Irlanda \\
\hline E CRS & 100,00 & 100,00 & 94,40 & 100,00 & 82,68 & 85,78 & 97,42 \\
\hline ECRS por pares & 35,38 & 71,88 & 71,98 & 63,89 & 54,21 & 5,17 & 61,54 \\
\hline País & Italia & Letonia & Lituania & Luxemburgo & Malta & Holanda & Polonia \\
\hline E CRS & 100,00 & 100,00 & 100,00 & 100,00 & 98,12 & 100,00 & 100,00 \\
\hline ECRS por pares & 70,83 & 2,73 & 6,66 & 95,21 & 62,76 & 70,68 & 32,83 \\
\hline País & Portugal & Rumania & Eslovaquia & Eslovenia & España & Suecia & Reino Unido \\
\hline E CRS & 74,67 & 100,00 & 93,59 & 100,00 & 100,00 & 68,55 & 99,19 \\
\hline ECRS por pares & 57,28 & 1,36 & 22,56 & 88,78 & 67,21 & 52,86 & 62,52 \\
\hline
\end{tabular}

Fuente: Elaboración propia

Gráfico 1

Medidas de eficiencia DEA-VRS y cross-eficiencia en el 2015

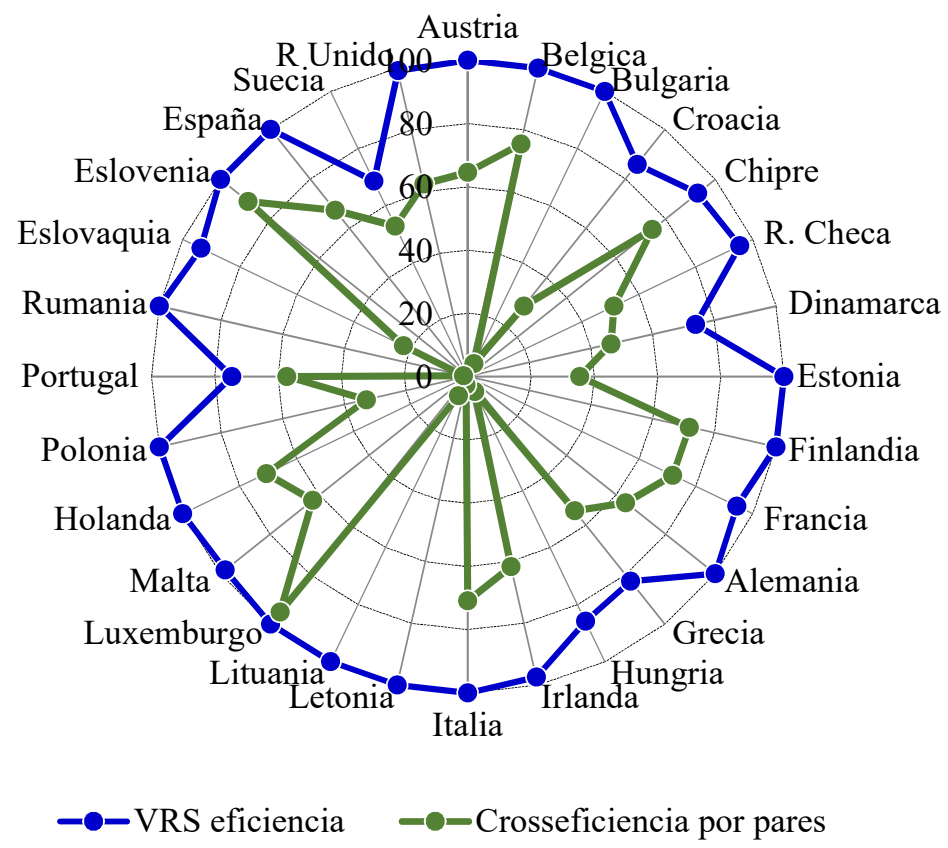

Fuente: Elaboración propia 


\subsection{Cambio en productividad}

En la tabla 4 se muestran los valores calculados del índice de Malmquist para el periodo 2012 a 2015. Asimismo, se presentan indicadores del cambio en eficiencia y el cambio en la tecnología de producción. Los índices estimados en este estudio permiten la comparación de los 28 países de la UE en cuanto a productividad, eficiencia e innovación tecnológica, en la atención de la salud.

Tabla 4.

Cambios en productividad, eficiencia y tecnología

\begin{tabular}{|c|c|c|c|}
\hline & Indices de Malmquist & Cambio en eficiencia & Cambio de frontera \\
\hline Austria & 0.962 & 0.982 & 0.980 \\
\hline Bélgica & 0.990 & 1.000 & 0.990 \\
\hline Bulgaria & 1.021 & 1.013 & 1.008 \\
\hline Croacia & 0.918 & 0.962 & 0.954 \\
\hline Chipre & 1.026 & 1.021 & 1.005 \\
\hline R. Checa & 1.035 & 1.054 & 0.982 \\
\hline Dinamarca & 0.993 & 1.000 & 0.993 \\
\hline Estonia & 1.034 & 1.025 & 1.009 \\
\hline Finlandia & 1.022 & 1.081 & 0.946 \\
\hline Francia & 0.987 & 1.017 & 0.971 \\
\hline Alemania & 0.997 & 1.000 & 0.997 \\
\hline Grecia & 0.991 & 1.006 & 0.986 \\
\hline Hungría & 1.000 & 1.022 & 0.979 \\
\hline Irlanda & 0.999 & 1.014 & 0.985 \\
\hline Italia & 1.008 & 1.031 & 0.977 \\
\hline Letonia & 1.031 & 1.101 & 0.936 \\
\hline Lituania & 0.937 & 1.000 & 0.937 \\
\hline Luxemburgo & 1.001 & 1.000 & 1.001 \\
\hline Malta & 1.012 & 1.030 & 0.982 \\
\hline Países Bajos & 0.972 & 0.991 & 0.981 \\
\hline Polonia & 0.968 & 1.000 & 0.968 \\
\hline Portugal & 1.038 & 1.044 & 0.995 \\
\hline Rumania & 0.929 & 1.000 & 0.929 \\
\hline Eslovaquia & 0.994 & 1.010 & 0.984 \\
\hline Eslovenia & 0.981 & 1.000 & 0.981 \\
\hline España & 1.022 & 1.027 & 0.995 \\
\hline Suecia & 0.980 & 0.999 & 0.982 \\
\hline Reino Unido & 0.968 & 0.968 & 0.968 \\
\hline Promedios & 0.994 & 1.014 & 0.979 \\
\hline Std & 0.031 & 0.029 & 0.021 \\
\hline
\end{tabular}

Fuente: Elaboración propia

A partir de la información mostrada en la tabla 4 , se deduce que, en promedio, ha habido un pequeño decrecimiento de la productividad $(0,6 \%)$ y en 11 casos de los $28,(39 \%)$, ha habido un incremento en productividad. Croacia es el país que ha tenido un mayor decrecimiento de la productividad $(8,2 \%)$. Portugal ha sido el país cuya productividad más ha crecido $(3,8 \%)$. 
La variación en productividad es el resultado de la agregación de dos factores: cambio en eficiencia y cambio en la tecnología de producción. Mientras que la eficiencia ha crecido en un 1,4\%, por término medio, el cambio tecnológico ha sido negativo $(2,21 \%)$. Los sistemas sanitarios de la Unión Europea, en este periodo, se han gestionado algo mejor, pero han obviado el uso tecnológico, quizá, tal vez, por las secuelas de la reciente crisis económica. Croacia ha sido, de nuevo, el país con un decrecimiento superior en la medida de eficiencia $(3,8 \%)$. En cuanto a la innovación tecnológica en el periodo, son los países de nuevo ingreso en la Unión Europea los que experimentan mayor decrecimiento: Letonia, Lituania y Rumania.

El siguiente gráfico 2 muestra la posición de los países respecto a los componentes del cambio en productividad. En el gráfico se señalan los cuatro cuadrantes que definen cuatro tipos de países: aquellos que mejoran en eficiencia y en tecnología, los que mejoran en eficiencia, pero no en innovación tecnológica, los que empeoran en eficiencia, pero mejoran en tecnología y aquellos que empeoran en ambos conceptos.

\section{Gráfico 2}

Distribución de países según eficiencia e innovación tecnológica

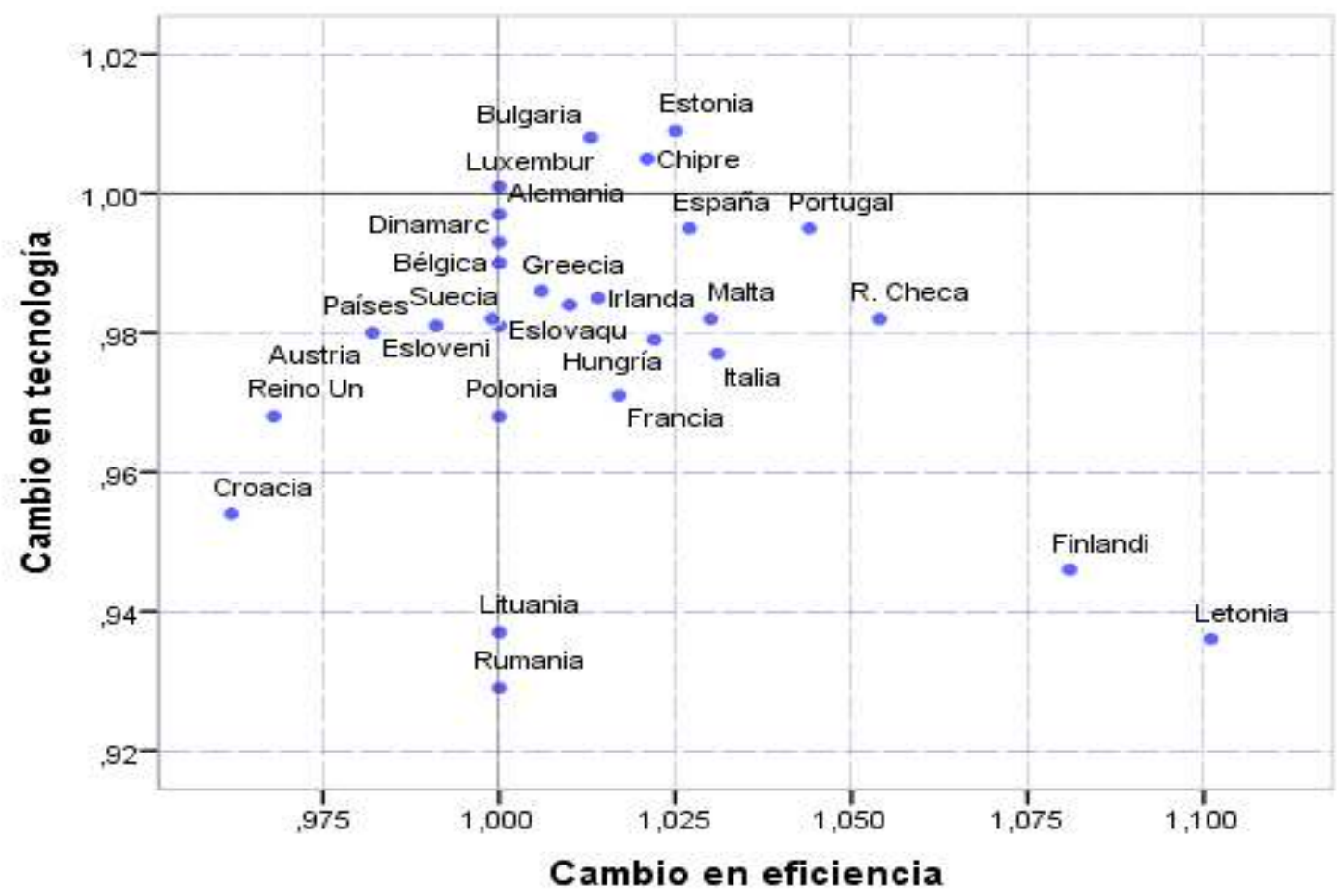

Fuente: Elaboración propia

Se observa en el gráfico 2 la heterogénea distribución de países por cuadrante, situándose en el tercer y cuarto cuadrante 24 de los 28 países europeos. Las posiciones extremas las ocupan Letonia, Estonia, Croacia y Rumanía.

En relación a las posibles causas asociadas a los cambios en productividad, De Vogli et al., (2005) y Leitner, (2015), argumentan que la desigualdad de ingresos en los países de la Unión Europea influye en algunos resultados de salud de la población, como por ejemplo en la esperanza de vida, la tasa de mortalidad infantil y las tasas de mortalidad estandarizadas por múltiples patologías. Una menor desigualdad de ingresos lleva a una mayor esperanza de vida y una menor mortalidad infantil. La OMS (2007) encontró que la esperanza de vida variaba incluso dentro de la clase de los países desarrollados, como evidencia de las desigualdades existentes entre ellos. Queremos resaltar con estos argumentos, la necesidad de realizar estudios adicionales a este trabajo con el fin de desentrañar y comprender las verdaderas causas de los resultados de salud de los respectivos sistemas sanitarios. El conocimiento de tales causas y de sus relaciones con los resultados de salud, permitirá diseñar e implementar estrategias de mejoras de eficiencia y de productividad. 


\section{CONCLUSIONES}

La estimación y análisis de la eficiencia en el periodo 2012 a 2015 en 28 países de La Unión Europea permite obtener las siguientes conclusiones:

- La gestión de los sistemas sanitarios europeos se realiza con considerables niveles de ineficiencia. Mejorar los niveles de eficiencia supondría disponer de recursos adicionales para alcanzar los deseables objetivos de los sistemas de salud.

- Entre los años 2012 y 2015 se ha producido una leve mejora en los niveles de eficiencia en la gestión de los sistemas de salud.

- El cambio en la tecnología de producción de salud ha sido negativo. Un muy alto porcentaje de países han decrecido en el nivel de la empleada tecnología sanitaria.

- El comportamiento de los países no es homogéneo. Los países clasificados como ineficientes podrían considerar las estrategias seguidas por sus países de referencia y valorar su imitación.

- En síntesis, los resultados de este estudio sugieren que las necesarias reformas políticas de los sistemas de salud pueden ajustarse para incrementar los niveles de eficiencia y mejorar en la innovación tecnológica sanitaria. Los países que no mejoran deben evaluar las estrategias prácticas de los sistemas de salud de los países pares, de sus referencias y desarrollar las políticas convenientes para lograr el crecimiento de la productividad en la provisión de salud de sus ciudadanos.

\section{REFERENCIAS BIBLIOGRÁFICAS}

AFONSO, A., AUBYN, M. (2005). "Non-parametric approaches to education and health efficiency in OECD countries". Journal of Applied Economics, 8(2), 227-246.

AFONSO, A., AUBYN, M. (2011). "Assessing health efficiency across countries with a two-step and bootstrap analysis". Applied Economics Letters, 18 (15), 1427-1430.

ANDERSON, T.R., HOLLINGSWORTH, K., INMAN, L. (2002). "The fixed weighting nature of a cross-evaluation model". Journal of Productivity Analysis, 17 (3), 249-255.

ANTOÑANZAS, F., JUÁREZ-CASTELLÓ C.A., RODRÍGUEZ-IBEAS, R. (2016). "Improving health care systems by building 'more Europe". European Journal of Health Economics, 17, 787-789.

ASANDULUIA, L., ROMANB, M., FATULESCUA, P. (2014). "The efficiency of healthcare systems in Europe: a data envelopment analysis approach". Procedia Economics and Finance, 10 (26), 261-268.

BALK, B.M. (2001). "Scale efficiency and productivity change". Journal of Productivity Analysis, 15 (3), 159-183.

BANKER, R.D., CHARNES, A., COOPER, W.W. (1984): "Some models for estimating technical and scale inefficiencies in Data Envelopment Analysis". Manage. Sci. 30, 1078-1092.

CHARNES, A., COOPER, W.W. (1984). "Some models for estimating technical and scale inefficiencies in Data Envelopment Analysis". Manage. Sci. 30, 1078-1092.

CAVES, D.W., CHRISTENSEN, L.R., DIEWERT W.E.(1982). "The economic theory of index numbers and the measurement of input, output, and productivity". Econometrica: J Econometric Soc., 1, 1393-1414.

CHARNES, A., COOPER, W.W., RHODES, E. (1978). "Measuring the efficiency of decision-making units". European Journal of Operational Research, 2, 429-444.

COOPER, W.W., SEIFORD, L.M., TONE, K. (2007). Data Envelopment Analysis, Springer.

DEBREU, G. (1951). "The Coefficient of Resource Utilization". Econométrica, 19 (3): 273-292.

DE VOGLI, R., MISTRY, R., GNESOTTO, R., CORNIA, G.A. (2005). "Has the relation between income inequality and life expectancy disappeared? Evidence from Italy and top industrialized countries". Journal of Epidemiology and Community Health, 59 (2), 158-162.

DOYLE, J., \& GREEN, R. (1994). "Efficiency and cross-efficiency in DEA: Derivations, meanings and uses". Journal of the operational research society, 45 (5), 567-578.

FARRELL, M.J. (1957). "The measurement of productive efficiency". Journal of the Royal Statistical Society. Series A, $120(3), 253-281$.

GEARHART, R. (2016). "No Theory: An Explanation of the Lack of Consistency in Cross-Country Health Care Comparisons Using Non-Parametric Estimators." Health Economics Review, 6, 40.

GEORGIOU, G. (2010). "The Budget of the European Union and its Contribution to Finance Programs for Health and Consumer Protection". European Planning Studies, 9 (8), 1031-1038.

GONZÁLEZ, E., CÁRCABA, A., VENTURA, J. (2010). "Value efficiency analysis of health systems: public financing does play a role?" Journal of Public Health, 18, 337-350.

GRIFELL-TATJÉ, E., LOVELL, C.A.K. (1997). "The Sources of Productivity Change in Spanish Banking". European Journal of Operational Research 98 (2), 364-380.

HERNÁNDEZ, P., MORAL-BENITO, E.(2014). "Determinants of health-system efficiency: evidence from OECD countries". International Journal of Health Economics and Management, 14, 69-93.

JACOBS, R, SMITH, P.C, STREET, A. (2006). "Measuring efficiency in health care: analytic techniques and health policy. Cambridge Univ. Pr. 
KOOPMANS, T.C. (1951). Analysis of production as an efficient combination of activities. In: Koopmans, T.C. (Eds.). Activity Analysis of Production and Allocation. Wiley, New York, 33-97.

LEITNER, S. (2015). "Effects of income inequality on publication health and social outcomes at the regional level in the EU". No. 113. The Vienna Institute for International Economic Studies, Wiener Institut für Internationale Wirtschaftsvergleiche.

LEWIN, A.Y. \& R.C. MOREY (1981). "Measuring the Output Potential of Public Sector Organizations: An Application of Data Envelopment Analysis". Int. J Policy Analysis and Information Systems, 5 (4): 267-285.

LIANG, L., WU, J., COOK, W. D., \& ZHU, J. (2008). "The DEA game cross-efficiency model and its Nash equilibrium". Operations research, 56 (5), 1278-1288.

MIRZOSAID, S. (2011). "Health expenditure efficiency in the Commonwealth of Independent States: A data envelopment analysis approach". Transition Studies Review, 18,384-404.

MORENO-ENGUIX, M.R., GÓMEZ-GALLEGO, J.C., GÓMEZ-GALLEGO, M. (2017). "Analysis and determination the efficiency of then European health systems". Int J Health Plann. Mgmt., 33, 136-154.

NUTI, S., DARAIO, C., SPERONI, C., VAINIERI, M. (2011). "Relationships between technical efficiency and the quality and costs of health care in Italy". International Journal for Quality in Health Care, 23(3), 324-330.

PÉREZ-CÁRCELES, M.C., GÓMEZ-GALLEGO, J.C., GÓMEZ- GALLEGO, M. (2017). "Environmental factors affecting European and Central Asian health-systems' bias-corrected efficiency". Applied Economics, 50 (32), 3432-3440.

QUAGLIO, G., KARAPIPERIS, T., VAN WOENSEL, L., ARNOLD, E.; MCDAID, D. (2013). Austerity and health in Europe. Health Policy, 113(1), 13-19.

RETZLAFF-ROBERTS, D., CHAN, C.F., RUBIN, R.M. (2004). "Technical efficiency in the use of health care resources: a comparison of OECD countries". Health Policy, 69, 55-72.

SAURINA, C., VALL-LLORESA, L., SÁEZ, M. (2012). "Factors determining access to and use of primary health care services in the Girona health region". European Journal of Health Economics, 13 (4), 419-427.

SEXTON, T.R., SILKMAN, R.H. \& HOGAN, A.J., (1986). "Data Envelopment Analysis: critique and extensions, en R.H. In: Silkman R.H. (ed.): Measuring efficiency: an assessment of Data Envelopment Analysis, Jossey-Bass, San Francisco. CA, 73-105.

SHEPHARD, R.W. (1953). Cost and production functions. Princeton.

SHEPHARD, R.W. (1970). Theory of cost and production functions.Princeton.

SIMAR, L., WILSON, P.W. (1998): "Sensitivity analysis of efficiency scores: how to bootstrap in nonparametric frontier models". Management Science, 44 (4), 49-61.

SIMAR, L., WILSON, P.W. (2007). "Estimation and inference in two-stage. Semi-parametric models of production processes". Journal of Econometrics, 136 (1), 31-64.

SORENSON, C., DRUMMOND, M., BHUIYAN KHAN, B. (2013). "Medical technology as a key driver of rising health expenditure: disentangling the relationship". Clinico economics and Outcomes Research, 5, 223-234.

SPINKS, J., HOLLINGSWORTH, B. (2009). "Cross-country comparisons of technical efficiency of health production: a demonstration of pitfalls". Applied Economics, 41, 417-427.

TUDOREL, A., MITRUT, C., CONSTANTIN, D., OANCEA, L. (2009). "The Impact of Decentralization on Public Health System's Results. The Case of Romania". Theoretical and Applied Economics, Asociatia Generala a Economistilor din Romania-AGER, 10(10(539)), 17-22.

VARABYOVA, Y., MÜLLER, J.M. (2016). "The efficiency of health care production in OECD countries: A systematic review and meta-analysis of cross-country comparisons". Health Policy, 120, 252-263. 
Anex01:

Valores de inputs y outputs para los años 2012 y 2015

\begin{tabular}{|c|c|c|c|c|c|c|c|c|c|c|c|c|}
\hline \multirow{2}{*}{ Países } & \multicolumn{3}{|c|}{ Inputs (2012) } & \multicolumn{3}{|c|}{ Outputs (2012) } & \multicolumn{3}{|c|}{ Inputs (2015) } & \multicolumn{3}{|c|}{ Outputs (2015) } \\
\hline & HEgdp & $\mathbf{P}$ & B & LE & ISR & HALE & HEgdp & $\mathbf{P}$ & B & LE & ISR & HALE \\
\hline Austria & 10,20 & 489,54 & 166,80 & 81,1 & 311,50 & 61,38 & 10,35 & 509,69 & 176,69 & 81,3 & 321,58 & 57,9 \\
\hline Bélgica & 10,11 & 292,80 & 189,34 & 80,5 & 262,16 & 64,61 & 10,09 & 301,75 & 193,09 & 81,1 & 302,03 & 64,4 \\
\hline Bulgaria & 7,59 & 391,45 & 178,70 & 74,4 & 127,21 & 63,95 & 8,20 & 404,54 & 166,40 & 74,7 & 150,52 & 61,5 \\
\hline Croacia & 6,59 & 299,18 & 256,97 & 77,3 & 276,78 & 63,81 & 7,15 & 319,15 & 279,23 & 77,5 & 242,90 & 63,1 \\
\hline Chipre & 6,69 & 301,64 & 288,94 & 81,1 & 284,71 & 63,17 & 6,81 & 359,34 & 292,80 & 81,8 & 369,37 & 62,4 \\
\hline R. Checa & 7,62 & 367,47 & 220,90 & 78,1 & 383,62 & 60,81 & 7,15 & 371 & 235,18 & 78,7 & 399,00 & 65,3 \\
\hline Dinamarca & 10,24 & 365,85 & 330,00 & 80,2 & 293,12 & 61,00 & 10,23 & 367,77 & 407,07 & 80,8 & 269,27 & 60,4 \\
\hline Estonia & 5,83 & 328,34 & 259,71 & 76,7 & 276,78 & 55,29 & 6,39 & 341,49 & 271,72 & 78 & 399,00 & 53,8 \\
\hline Finlandia & 9,29 & 306,87 & 307,63 & 80,7 & 415,67 & 60,42 & 9,74 & 325,00 & 327,49 & 81,6 & 587,24 & 63,9 \\
\hline Francia & 11,31 & 308,49 & 294,64 & 82,1 & 284,71 & 64,28 & 11,48 & 311,88 & 312,04 & 82,4 & 269,27 & 63,9 \\
\hline Alemania & 10,77 & 387,38 & 163,38 & 80,7 & 302,03 & 57,61 & 11,06 & 413,93 & 163,59 & 80,7 & 302,03 & 59,4 \\
\hline Greecia & 8,88 & 592,00 & 274,68 & 80,7 & 343,83 & 63,77 & 8,15 & 610,00 & 277,47 & 81,1 & 249,00 & 62,6 \\
\hline Hungría & 7,47 & 308,87 & 232,06 & 75,3 & 203,08 & 61,17 & 7,11 & 309,72 & 233,82 & 75,7 & 237,10 & 55,3 \\
\hline Irlanda & 10,79 & 271,43 & 421,40 & 80,9 & 284,71 & 63,89 & 7,43 & 287,5 & 417,16 & 81,5 & 293,12 & 58,2 \\
\hline Italia & 8,96 & 387,34 & 351,51 & 82,4 & 343,83 & 63,63 & 8,99 & 383,83 & 379,36 & 82,7 & 343,83 & 66,6 \\
\hline Letonia & 5,44 & 314,40 & 280,91 & 74,1 & 157,73 & 60,42 & 5,71 & 319,79 & 295,15 & 74,8 & 242,90 & 62,6 \\
\hline Lituania & 6,29 & 421,85 & 154,37 & 74,1 & 255,41 & 59,30 & 6,48 & 433,92 & 164,39 & 74,6 & 237,10 & 54,1 \\
\hline Luxemburgo & 6,57 & 278,37 & 252,71 & 81,5 & 399,00 & 66,10 & 6,25 & 290,73 & 248,09 & 82,4 & 356,14 & 63,7 \\
\hline Malta & 6,50 & 329,24 & 400,63 & 80,9 & 187,68 & 63,45 & 6,30 & 378,81 & 308,48 & 82 & 171,41 & 51,8 \\
\hline Países Bajos & 10,77 & 340,00 & 273,89 & 81,2 & 269,27 & 65,14 & 10,30 & 348,72 & 290 & 81,6 & 302,03 & 72,6 \\
\hline Polonia & 6,42 & 223,38 & 201,72 & 76,9 & 216,39 & 63,14 & 6,34 & 232,81 & 203,55 & 77,5 & 249,00 & 61,1 \\
\hline Portugal & 9,35 & 432,00 & 299,35 & 80,6 & 293,12 & 60,93 & 8,97 & 440,00 & 309,35 & 81,3 & 343,83 & 60,1 \\
\hline Rumania & 4,72 & 261,05 & 204,46 & 74,4 & 110,11 & 61,01 & 4,94 & 276,59 & 198,74 & 75 & 130,58 & 58,2 \\
\hline Eslovaquia & 7,63 & 325,00 & 198,97 & 76,3 & 171,41 & 55,29 & 6,85 & 339 & 205,13 & 76,7 & 195,08 & 59,0 \\
\hline Eslovenia & 8,18 & 254,14 & 233,48 & 80,3 & 624,00 & 60,74 & 8,50 & 282,53 & 237,24 & 80,9 & 624,00 & 74,0 \\
\hline España & 9,08 & 382,34 & 420,50 & 82,5 & 321,58 & 61,21 & 9,11 & 384,52 & 415,30 & 83 & 369,37 & 58,5 \\
\hline Suecia & 10,93 & 404,67 & 414,10 & 81,8 & 383,62 & 59,72 & 11,00 & 427,06 & 441,96 & 82,2 & 399,00 & 54,8 \\
\hline Reino Unido & 9,48 & 269,62 & 280,00 & 81 & 249,00 & 64,55 & 9,76 & 277,06 & 300 & 81 & 255,41 & 63,7 \\
\hline
\end{tabular}


Anexo2:

Tasa de variación de inputs y outputs

\begin{tabular}{|c|c|c|c|c|c|c|}
\hline \multirow{2}{*}{ Países } & \multicolumn{6}{|c|}{ Tasa de variación (\%) } \\
\hline & HEgdp & $\mathbf{P}$ & B & LE & ISR & HALE \\
\hline Austria & 1,48 & 4,12 & 5,93 & 0,25 & 3,24 & $-5,67$ \\
\hline Bélgica & $-0,18$ & 3,06 & 1,98 & 0,75 & 15,21 & $-0,32$ \\
\hline Bulgaria & 8,00 & 3,34 & $-6,88$ & 0,40 & 18,32 & $-3,83$ \\
\hline Croacia & 8,50 & 6,67 & 8,66 & 0,26 & $-12,24$ & $-1,12$ \\
\hline Chipre & 1,80 & 19,13 & 1,34 & 0,86 & 29,73 & $-1,22$ \\
\hline R. Checa & $-6,21$ & 0,96 & 6,46 & 0,77 & 4,01 & 7,38 \\
\hline Dinamarca & $-0,14$ & 0,52 & 23,35 & 0,75 & $-8,14$ & $-0,99$ \\
\hline Estonia & 9,59 & 4,00 & 4,62 & 1,69 & 44,16 & $-2,69$ \\
\hline Finlandia & 4,86 & 5,91 & 6,46 & 1,12 & 41,28 & 5,75 \\
\hline Francia & 1,49 & 1,10 & 5,91 & 0,37 & $-5,42$ & $-0,60$ \\
\hline Alemania & 2,72 & 6,85 & 0,13 & 0,00 & 0,00 & 3,11 \\
\hline Grecia & $-8,24$ & 3,04 & 1,02 & 0,50 & $-27,58$ & $-1,84$ \\
\hline Hungría & $-4,78$ & 0,28 & 0,76 & 0,53 & 16,75 & $-9,59$ \\
\hline Irlanda & $-31,13$ & 5,92 & $-1,01$ & 0,74 & 2,95 & $-8,91$ \\
\hline Italia & 0,36 & $-0,91$ & 7,92 & 0,36 & 0,00 & 4,67 \\
\hline Letonia & 4,97 & 1,71 & 5,07 & 0,94 & 54,00 & 3,61 \\
\hline Lituania & 2,98 & 2,86 & 6,49 & 0,67 & $-7,17$ & $-8,76$ \\
\hline Luxemburgo & $-4,86$ & 4,44 & $-1,83$ & 1,10 & $-10,74$ & $-3,63$ \\
\hline Malta & $-3,08$ & 15,06 & $-23,00$ & 1,36 & $-8,67$ & $-18,36$ \\
\hline Países Bajos & $-4,43$ & 2,56 & 5,88 & 0,49 & 12,17 & 11,46 \\
\hline Polonia & $-1,25$ & 4,22 & 0,91 & 0,78 & 15,07 & $-3,23$ \\
\hline Portugal & $-4,03$ & 1,85 & 3,34 & 0,87 & 17,30 & $-1,36$ \\
\hline Rumania & 4,77 & 5,95 & $-2,80$ & 0,81 & 18,59 & $-4,60$ \\
\hline Eslovaquia & $-10,31$ & 4,31 & 3,10 & 0,52 & 13,81 & 6,71 \\
\hline Eslovenia & 3,90 & 11,17 & 1,61 & 0,75 & 0,00 & 21,82 \\
\hline España & 0,38 & 0,57 & $-1,24$ & 0,61 & 14,86 & $-4,43$ \\
\hline Suecia & 0,71 & 5,53 & 6,73 & 0,49 & 4,01 & $-8,24$ \\
\hline Reino Unido & 2,96 & 2,76 & 7,14 & 0,00 & 2,57 & $-1,32$ \\
\hline
\end{tabular}


Anexo 3:

Conjuntos de referencia en el año 2012

\begin{tabular}{|c|c|c|c|c|c|c|c|c|c|c|c|}
\hline \multirow{2}{*}{\begin{tabular}{l}
\multicolumn{1}{c}{ Países } \\
Austria
\end{tabular}} & \multirow{2}{*}{$\frac{\text { Siglas }}{\text { AT }}$} & \multicolumn{10}{|c|}{ Conjuntos de países de referencia } \\
\hline & & 1 & AT & & & & & & & & \\
\hline Bélgica & $\mathrm{BE}$ & 1 & $\mathrm{BE}$ & & & & & & & & \\
\hline Bulgaria & BG & 1 & BG & & & & & & & & \\
\hline Croacia & HR & 0,003 & LT & 0,515 & LU & 0,09 & $\mathrm{PL}$ & 0,376 & RO & 0,015 & SI \\
\hline Chipre & $\mathrm{CY}$ & 0,944 & LU & 0,056 & RO & & & & & & \\
\hline R. Checa & $C Z$ & 0,048 & $\mathrm{DE}$ & 0,417 & LT & 0,312 & LU & 0,003 & PL & 0,221 & SI \\
\hline Dinamarca & DK & 0,031 & $\mathrm{BE}$ & 0,408 & LU & 0,175 & $\mathrm{PL}$ & 0,385 & SI & & \\
\hline Estonia & EE & 0,577 & LU & 0,423 & RO & & & & & & \\
\hline Finlandia & $\mathrm{FI}$ & 0,333 & LU & 0,667 & SI & & & & & & \\
\hline Francia & FR & 1 & FR & & & & & & & & \\
\hline Alemania & DE & 1 & $\mathrm{DE}$ & & & & & & & & \\
\hline Grecia & GR & 0,206 & AT & 0,097 & LT & 0,697 & LU & & & & \\
\hline Hungría & $\mathrm{HU}$ & 0,161 & LT & 0,752 & PL & 0,086 & RO & & & & \\
\hline Irlanda & IE & 0,098 & LU & 0,213 & SI & 0,69 & GB & & & & \\
\hline Italia & IT & 1 & IT & & & & & & & & \\
\hline Letonia & LV & 0,165 & LU & 0,835 & RO & & & & & & \\
\hline Lituania & LT & 1 & LT & & & & & & & & \\
\hline Luxemburgo & LU & 1 & LU & & & & & & & & \\
\hline Malta & MT & 0,915 & LU & 0,085 & RO & & & & & & \\
\hline Países Bajos & NL & 0,259 & $\mathrm{BE}$ & 0,051 & $\mathrm{DE}$ & 0,69 & LU & & & & \\
\hline Polonia & PL & 1 & PL & & & & & & & & \\
\hline Portugal & PT & 0,159 & AT & 0,113 & LT & 0,728 & LU & & & & \\
\hline Rumania & RO & 1 & RO & & & & & & & & \\
\hline Eslovaquia & SK & 0,078 & $\mathrm{DE}$ & 0,32 & LT & 0,601 & PL & & & & \\
\hline Eslovenia & SI & 1 & SI & & & & & & & & \\
\hline España & ES & 1 & ES & & & & & & & & \\
\hline Suecia & SE & 0,569 & LU & 0,059 & SI & 0,371 & ES & & & & \\
\hline Reino Unido & GB & 1 & GB & & & & & & & & \\
\hline
\end{tabular}


Anexo 4:

Conjuntos de referencia en el año 2015

\begin{tabular}{|c|c|c|c|c|c|c|c|c|c|}
\hline Países & Siglas & & & juuntos & le pa & es de & feren & & \\
\hline Austria & AT & 1,000 & AT & & & & & & \\
\hline Bélgica & BE & 1,000 & $\mathrm{BE}$ & & & & & & \\
\hline Bulgaria & $B G$ & 1,000 & BG & & & & & & \\
\hline Croacia & HR & 0,093 & LU & 0,122 & $\mathrm{PO}$ & 0,529 & RO & 0,255 & $\mathrm{SI}$ \\
\hline Chipre & $\mathrm{CY}$ & 0,126 & EE & 0,844 & LU & 0,029 & SI & & \\
\hline R. Checa & $\mathrm{CZ}$ & 0,155 & LU & 0,372 & RO & 0,473 & SI & & \\
\hline Dinamarca & DK & 0,673 & LU & 0,327 & $\mathrm{PO}$ & & & & \\
\hline Estonia & EE & 1,000 & EE & & & & & & \\
\hline Finlandia & $\mathrm{FI}$ & 1,000 & $\mathrm{FI}$ & & & & & & \\
\hline Francia & FR & 0,032 & IT & 0,956 & LU & 0,012 & $\mathrm{NL}$ & & \\
\hline Alemania & $\mathrm{DE}$ & 1,000 & $\mathrm{DE}$ & & & & & & \\
\hline Grecia & GR & 0,097 & $\mathrm{DE}$ & 0,015 & $\mathrm{LT}$ & 0,680 & $\mathrm{LU}$ & 0,208 & $\mathrm{PO}$ \\
\hline Hungría & $\mathrm{HU}$ & 0,091 & $\mathrm{LT}$ & 0,585 & $\mathrm{PO}$ & 0,268 & RO & 0,056 & $\mathrm{SI}$ \\
\hline Irlanda & IE & 0,816 & LU & 0,184 & $\mathrm{PO}$ & & & & \\
\hline Italia & IT & 1,000 & IT & & & & & & \\
\hline Letonia & LV & 1,000 & LV & & & & & & \\
\hline Lituania & $\mathrm{LT}$ & 1,000 & $\mathrm{LT}$ & & & & & & \\
\hline Luxemburgo & LU & 1,000 & LU & & & & & & \\
\hline Malta & MT & 0,946 & LU & 0,054 & RO & & & & \\
\hline Países Bajos & $\mathrm{NL}$ & 1,000 & $\mathrm{NL}$ & & & & & & \\
\hline Polonia & PL & 1,000 & $\mathrm{PO}$ & & & & & & \\
\hline Portugal & PT & 0,091 & AT & 0,124 & $\mathrm{LT}$ & 0,764 & $\mathrm{LU}$ & 0,021 & $\mathrm{SI}$ \\
\hline Rumania & RO & 1,000 & $\mathrm{RO}$ & & & & & & \\
\hline Eslovaquia & SK & 0,006 & $\mathrm{DE}$ & 0,303 & $\mathrm{LT}$ & 0,012 & LU & 0,679 & $\mathrm{PO}$ \\
\hline Eslovenia & SI & 1,000 & $\mathrm{SI}$ & & & & & & \\
\hline España & ES & 1,000 & ES & & & & & & \\
\hline Suecia & SE & 0,081 & $\mathrm{FI}$ & 0,829 & LU & 0,090 & SI & & \\
\hline Reino Unido & GB & 0,668 & LU & 0,265 & $\mathrm{PO}$ & 0,067 & $\mathrm{SI}$ & & \\
\hline
\end{tabular}

